\title{
直写成型用悬浮液的设计
}

\author{
王小锋 ${ }^{1}$, 孙月花 ${ }^{1}$, 彭超群 ${ }^{1}$, 王日初 ${ }^{1}$, 张 ${ }^{2}$, 马 超 ${ }^{2}$ \\ (中南大学 1. 材料科学与工程学院; 2.粉末冶金国家重点实验室, 长沙 410083)
}

\begin{abstract}
摘 要: 直写成型技术是一种新型的三维复杂结构的制备方法。本文综述了直写成型用悬浮液的研究进展: 根据直 写成型悬浮液的固化特点, 将之划分为自固化悬浮液和外固化悬浮液; 分析了自固化悬浮液的流变性能要求与设 计准则, 综述了典型的自固化悬浮液; 分析了外固化悬浮液的要求与固化方式, 并总结了典型的外固化悬浮液与 固化方式; 探讨了直写成型用悬浮液的发展方向。
\end{abstract}

关 键 词: 直写成型; 悬浮液; 设计; 三维结构; 综述

中图分类号: TQ123 文献标识码: A

\section{Suspensions Designed for Direct Ink Writing}

\author{
WANG Xiao-Feng ${ }^{1}$, SUN Yue-Hua ${ }^{1}$, PENG Chao-Qun ${ }^{1}$, WANG Ri-Chu ${ }^{1}$, ZHANG Dou $^{2}$, MA Chao ${ }^{2}$ \\ (1. College of Materials Science and Engineering, Central South University, Changsha 410083, China; 2. State Key Laboratory \\ of Powder Metallurgy, Central South University, Changsha 410083, China)
}

\begin{abstract}
Direct ink writing is a novel route for three-dimensional complex structures. Research progress of suspensions for direct ink writing is reviewed in this paper. These suspensions are divided to self- and assistant-solidification suspensions according to their solidification feature. Rheological characterizations and design principles of self-solidification suspensions are analyzed, and typical suspensions are concluded. Design principles and solidification mode of assistant-solidification suspensions are also analyzed, including typical assistant-solidification suspensions and solidification mode. Finally, the research tendency of suspensions for direct ink writing is discussed.
\end{abstract}

Key words: direct ink writing; suspension; design; three-dimensional structure; review

三维周期结构已广泛地用于先进陶瓷材料 ${ }^{[1-6]}$ 、 复合材料 ${ }^{[7-10]}$ 、组织工程支架 ${ }^{[11-13]}$ 和光子晶体 ${ }^{[14-16]}$ 等的设计和制备。直写成型(Direct ink writing/DIW,

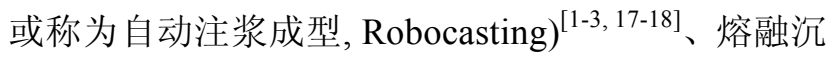
积成型(Fused deposition) ${ }^{[4,19-21]}$ 和三维打印成型(3D printing $)^{[22-24]}$ 等技术也被越来越多地用于三维周期 结构的成型。其中, 直写成型的三维结构具有很大 高宽比和尺寸控制范围 (从 $10^{-6}$ 到 $10^{0} \mathrm{~m}$ ) 等优点, 并 且可成型含跨度(无支撑部分)的复杂三维结构, 所 以该方法受到广泛关注 ${ }^{[1-3,25-27]}$ 。根据成型基本单元
的不同，直写成型可分为通过逐滴堆积的滴形直写 成型和采用连续线形叠加的线形直写成型 ${ }^{[3]}$ 。本文 所述直写成型主要指线形直写成型。

直写成型的概念首先由美国桑迪亚国家实验室 的 Cesarano 等 ${ }^{[17]}$ 提出，其示意图如图 1 所示 ${ }^{[1,25-26]}$ 。 该技术首先借助计算机辅助(CAD)设计所需的三维 结构, 然后通过计算机自动控制安装在 $\mathrm{Z}$ 轴上的悬 浮液输送装置将之精确输出形成特征线形流体(图 $1(\mathrm{~b})$ 和(c)), 同时 $\mathrm{X}-\mathrm{Y}$ 轴依照程序设定的轨迹移动, 将线形流体沉积在运动平台上, 得到第一层结构。

收稿日期：2015-04-03; 收到修改稿日期：2015-05-27

基金项目：国家青年自然科学基金(51202296); 高等学校博士学科点专项科研基金(20120162120006)

National Natural Science Foundation of China (51202296); Specialized Research Fund for the Doctoral Program of Higher Education (20120162120006)

作者简介: 王小锋(1980-), 男, 博士. E-mail: wangxiaofeng@csu.edu.cn

通讯作者: 王日初, 教授. E-mail: 13467516329@163.com 

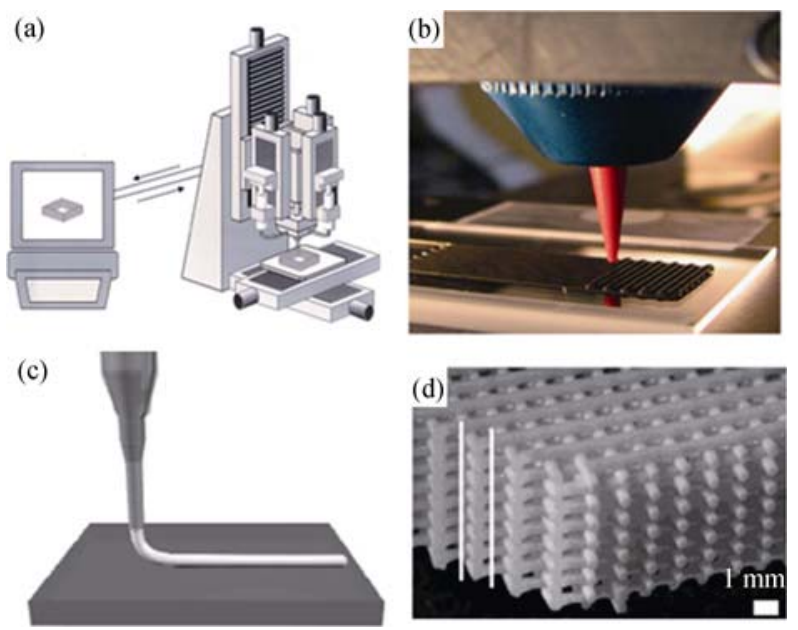

图 1 直写成型(自动注浆成型)示意图

Fig. 1 Direct ink writing (robocasting)

(a) Schematic view ${ }^{[1]}$ and (b) optical image of direct ink writing ${ }^{[26]}$; (c) schematic view of filament fluid ${ }^{[1]}$; (d) optical image of a 3-D periodic structure ${ }^{[25]}$

随后, $\mathrm{Z}$ 轴马达带动悬浮液输送装置精确地向上移 动到设定高度, 在第一层结构上进行第二层成型。 最后, 通过逐层叠加的方式, 获得传统工艺无法制 备的复杂三维结构(图 1(d))。

悬浮液的成分、微观组织结构和流变性能的设 计与控制是直写成型的重点和难点 ${ }^{[1-3]}$, 学者们对 此进行了大量的研究, 其中美国伊利诺伊大学厄巴 纳-香槟分校的 Lewis 研究组在直写成型的悬浮液 设计与制备等方面开展了许多原创性的工作, 推动 了直写成型的发展。最初, Cesarano 等 ${ }^{[28-29]}$ 采用具有 高剪切变稀流变性能的胶体悬浮液并通过外界条件 控制溶剂蒸发速率的方式来进行直写成型。Smay 等 ${ }^{[25}$ 设计了一种能够很好满足直写成型要求的胶 体凝胶悬浮液, 并将其用于直写成型。该悬浮液的 微观组织为互相吸引的颗粒组成的网络结构, 所以 当固相体积分数高于其凝胶点 $\left(\Phi_{\mathrm{gel}}\right.$, 悬浮液表现为 凝胶态所对应的固相体积分数) 时, 悬浮液能够在 压力作用下较顺利地从悬浮液输送装置(如针头)中 流出, 并且在压力消失之后其自身的流动性降低且 粘弹性急剧升高, 因而能够承受一定的重力而不发 生变形。Smay 等 ${ }^{[25]}$ 研究的悬浮液是通过自身流变性 能的变化而固化的, 解决制约直写成型需依靠外界 条件使溶剂蒸发才能固化的瓶颈问题。Gratson 等 ${ }^{[30]}$ 研究了由聚乙烯亚胺(PEI)和聚丙烯酸(PAA)组成的 聚电解质混合体悬浮液, 并通过外界条件的变化, 即悬浮液在其他溶剂中的溶解差异等实现直写成 型。虽然针对直写成型悬浮液设计和成型的研究较 多, 但尚缺乏系统的总结。

本文主要综述直写成型悬浮液的设计: 根据直
写成型悬浮液(线形特征流体)的固化特点, 将悬浮 液分为自固化悬浮液和外固化悬浮液两种; 分析自 固化悬浮液的要求与设计准则, 综述典型的自固化 悬浮液; 分析外固化悬浮液的要求与固化方式, 并 总结典型的外固化悬浮液与固化方式; 最后探讨直 写成型悬浮液的发展方向。

\section{1 直写成型悬浮液的类型}

在成型过程中, 直写成型首先采用特殊的装置 (例如针嘴微孔)使悬浮液形成线形的特征流体, 然 后通过计算机自动控制线形特征流体以及承接特征 流体的运动平台的移动轨迹, 从而制备预定结构和 成分的复杂结构 ${ }^{[3]}$ 。显然, 为了获得具有三维立体特 征的复杂周期结构, 线形特征流体离开针孔等特殊 装置之后, 即可以通过悬浮液自身性能的转变(如 流变性能变化 ${ }^{[25]}$ )来实现快速固化(失去流动性), 也 可以通过外界条件的作用 (如溶剂蒸发 ${ }^{[28]}$ 或紫外线 固化 ${ }^{[31]}$ 等)使悬浮液结构发生变化来实现快速固化, 并保持自身形状不变并支撑后续成型的线形特征流 体。因此，根据线形特征流体失去流动性的 “固化” 情况可将直写成型划分为两大类：自固化直写成型 和外固化直写成型。相应地, 我们将悬浮液分为依 靠自身流变性能转变 “自行固化” 的悬浮液(简称自 固化悬浮液或自支撑型悬浮液)和借助外界条件作 用使其微观组织结构变化进行固化的悬浮液(简称 外固化悬浮液)两类。

\section{2 自固化悬浮液的设计}

\section{1 自固化悬浮液的要求}

直写成型对自固化悬浮液的要求很高。它要求 悬浮液必须能够顺利通过特征流体生成装置(针孔) 形成线形流体，并以快速固化的方式保持形状，且 获得的悬空梁结构不发生弯曲和坡塌等变形。因此, 自固化悬浮液必需满足四个基本条件 ${ }^{[3,25]}$ : 1)高剪 切条件下粘度较低, 才能够顺利通过针嘴微孔而不 发生堵塞；2)无剪切条件下失去流动性，即通过针 嘴微孔之后的线形流体必须快速固化; 3)固化后有 很好的弹性性能; 4)高固相体积分数, 在干燥(或烧 结)等后处理程序中, 可以避免成型的三维复杂结 构发生变形和开裂。总的来说, 直写成型要求悬浮 液在通过针嘴微孔时具有较好的流动性, 并在通过 之后具有很好的弹性。可见, 普通的悬浮液并不能 满足自固化直写成型三维结构的要求 ${ }^{[17,25]}$ 。 


\subsection{1 悬浮液的流变性能}

在直写成型过程中, 悬浮液通过针嘴微孔时的 流动情况很复杂。悬浮液进入微孔之后形成线形流 体, 该线形流体的表面在与微孔内壁之间产生的摩 擦力的剪切作用下发生流动, 内部的悬浮液则保持 凝胶特性而基本不发生流动, 所以线形特征流体在 通过微孔时的结构是一种(刚性凝胶态)核-(剪切流 动态) 壳结构 ${ }^{[25]}$ 。需指出的是核一壳之间没有十分清 晰的界限。因此, 只有当线形特征流体表面的悬浮 液(剪切流动态壳) 表现出剪切变稀流变行为时, 线 形特征流体才能顺利地通过微孔, 此类悬浮液的流 变性能很好地满足 Herschel-Bulkley 模型 ${ }^{[31-32]}$ :

$$
\tau=\tau_{y}+K \gamma^{n}
$$

其中, $\tau$ 是剪切应力, $\tau_{y}$ 是剪切屈服应力, $K$ 是粘度指 数, $n$ 是剪切变稀指数, $\gamma$ 是剪切速率。当悬浮液受到 的剪切应力 $(\tau)$ 高于剪切屈服应力 $\left(\tau_{y}\right)$ 时, 颗粒间的 链接作用被破坏, 表面悬浮液才能发生流动。在整 个成型过程中, 线形特征流体内部的悬浮液(刚性 凝胶态核) 性能基本不变, 能够起到保持成型形状 的作用, 并支撑具有跨度部分的自身重量。但是如果 悬浮液受到的剪切应力大于压缩屈服应力 $\left(P_{y}\right)$, 线形 特征流体内部的悬浮液将出现颗粒与溶剂分离的压 滤现象 ${ }^{[25]}$, 堵塞针嘴微孔。所以剪切应力 $(\tau)$ 必须低于 悬浮液的压缩屈服应力 $\left(P_{y}\right)$ 。可见, 在直写成型过程 中, 施加的剪切应力 $(\tau)$ 的范围是: $\tau_{y}<\tau<P_{y}$ 。

\subsection{2 悬浮液的弹性性能}

线形特征流体流出微孔之后不再受到剪切应力 的作用, 剪切流动态壳内的颗粒之间重新发生链接, 悬浮液转变为刚性凝胶态, 起到与刚性凝胶态核相同 的作用。凝胶态的弹性性能可用下式表示 ${ }^{[27,33]}$.

$$
y=\mathrm{k}\left(\frac{\Phi}{\Phi_{\text {gel }}}-1\right)^{x}
$$

其中, $y$ 为悬浮液的弹性性能(包括剪切屈服应力 $\tau_{y}$ 和弹性模量 $\left.G^{\prime}\right), \mathrm{k}$ 为常数, $\Phi$ 为固相体积分数, $\Phi_{\mathrm{gel}}$ 是凝胶临界点对应的固相体积分数, $x$ 是比例常数 (一般为 2.5 左右)。可见, 凝胶的弹性性能主要由两 个参数分别为固相体积分数 $(\Phi)$ 和凝胶固相体积分 数 $\left(\Phi_{\mathrm{gel}}\right)$ 决定。随着 $\Phi$ 的增加, 悬浮液中颗粒间链接 键的密度增大, 弹性性能升高。而 $\Phi_{\mathrm{gel}}$ 的作用则相反, $\Phi_{\text {gel }}$ 的增加会使链接键的强度降低, 弹性性能降低。 因此, 为了满足直写成型的要求, 胶体悬浮液的弹 性性能可通过调节 $\Phi$ 和 $\Phi_{\mathrm{gel}}$ 来实现。

\section{2 自固化悬浮液的设计准则}

自固化悬浮液(胶体凝胶悬浮液)内的颗粒均处
于相互吸引的状态, 其流变性能主要由承受的应力 $(\sigma)$ 、固相体积分数 $(\Phi)$ 和颗粒间相互吸引作用能 $(U)$ 三个参数决定, 如图 2 所示 ${ }^{[34]}$ 。悬浮液承受的应力 对颗粒间相互吸引形成的网络结构存在影响, 并且 临界固相体积分数随着应力的增大而升高。但是在 直写成型中, 对悬浮液施加的应力为成型工艺参数, 不能单独用于调节流变性能。如前所述，固相体积 分数的增加可以使悬浮液的剪切屈服应力和弹性模 量升高(式(2)), 并且悬浮液的固相体积分数本应尽 可能的高, 所以固相体积分数也不能用于设计自固 化悬浮液。而悬浮液内颗粒间相互作用受多种因素 的影响，可以很容易地通过调控颗粒间的相互作用， 使颗粒间的相互吸引作用能增强，从而达到满足直 写成型要求的自固化悬浮液的目的。

颗粒在悬浮液中受到的作用主要有范德华引力 作用、静电排斥作用、空间位阻作用和耗散作用 ${ }^{[1]}$ 。 假设吸引作用能为正值, 排斥作用能为负值, 则颗 粒间的相互作用能可以表示为:

$$
U_{\text {total }}=U_{\mathrm{vdW}}+U_{\text {elect }}+U_{\text {steric }}+U_{\text {depletion }}
$$

其中, $U_{\mathrm{vdW}}$ 为颗粒间长程范德华作用产生的吸引作 用能(取为正值); $U_{\text {elect }}$ 为带电颗粒相互作用产生的 静电排斥能(取为负值); $U_{\text {steric }}$ 为颗粒表面吸附聚合 物而产生的位阻作用能(增加悬浮液的稳定性, 取 为负值); $U_{\text {depletion }}$ 为悬浮液中非吸附聚合物产生的 耗散作用能(增加(取为负值)或降低(或取为正值)悬 浮液的稳定性)。因此, 调节某些条件使 $U_{\mathrm{vdW}}$ 、

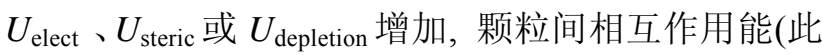
时即为相互吸附作用能)增加, 从而获得满足成型 要求的自固化悬浮液。这些条件包括 $\mathrm{pH}$ 、盐浓度、

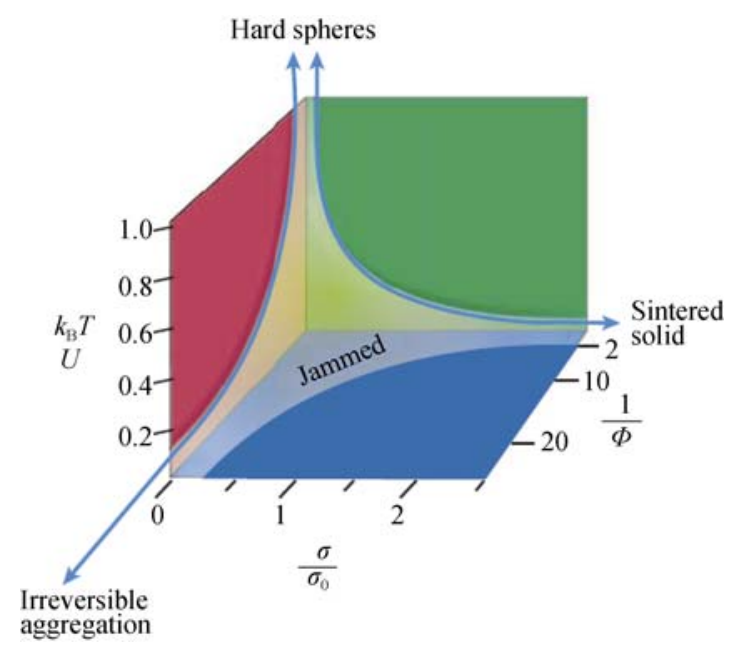

图 2 相互吸引的胶态颗粒的聚集状态图 ${ }^{[34]}$

Fig. 2 Jamming phase diagram for attractive colloidal particles $^{[34]}$ 
反离子聚电解质的加入、聚电解质分散剂的种类及 其溶剂的性质等等。前三者已经用来设计并制备自 固化悬浮液 ${ }^{[13,25,35-39]}$, 而通过其他条件设计自固化 悬浮液尚未见相关报道。

\section{3 典型的自固化悬浮液}

目前采用的自固化悬浮液均为通过某种手段调 控悬浮液流变性能的胶体悬浮液, 主要有胶体凝胶 悬浮液和双相悬浮液两大类, 如表 1 所列。

\subsection{1 胶体凝胶悬浮液}

最初用于直写成型的悬浮液为剪切变稀胶体悬 浮液，但它不能用于制备具有跨度(无支撑部分)的 三维结构, 因其弹性性能不够, 不能够支撑自身的
重力。为了解决此问题, Smay 等 ${ }^{[25]}$ 设计了胶体凝胶 悬浮液, 即在溶剂中胶体颗粒发生絮凝并相互链接 的悬浮液，他首先制备稳定分散的胶体悬浮液，然 后通过改变悬浮液的酸碱度，使胶体颗粒发生聚集， 从而获得亚微米级锆钛酸铅 $(\mathrm{PZT})$ 粉体的胶体凝胶 悬浮液, 并成型出三维结构。此外, 通过改变悬浮液 的离子浓度 ${ }^{[37]}$ 或加入反离子聚电解质 ${ }^{[38]}$ 等方式也 可以获得胶体凝胶悬浮液。上述三种方法均是使流 动态悬浮液中的胶体颗粒发生絮凝，从而降低悬浮 液的凝胶点固相体积分数, 增加悬浮液的弹性性能, 最终形成凝胶态的胶体悬浮液。图 3 所示为胶体悬 浮液从流动态到凝胶态的结构转变示意图 ${ }^{[25,36-38]}$ 。

表 1 直写成型的自固化悬浮液 ${ }^{[13,25,35-39]}$

Table 1 Self-solidification suspensions designed for direct ink writing ${ }^{\text {[13, 25, 35-39] }}$

\begin{tabular}{|c|c|c|c|}
\hline $\begin{array}{l}\text { Self-solidification } \\
\text { suspensions }\end{array}$ & $\begin{array}{l}\text { Tailoring routes for rheological } \\
\text { properties of suspensions }\end{array}$ & $\begin{array}{l}\text { Minimum feature size in } \\
\text { 3D structures }\end{array}$ & References \\
\hline \multirow[t]{3}{*}{ Colloidal gel suspensions } & Changing $\mathrm{pH}$ values & $100 \mu \mathrm{m}$ & {$[25,35-36]$} \\
\hline & Tailoring ionic concentrations & $30 \mu \mathrm{m}$ & {$[37]$} \\
\hline & Adding oppositive polyelectrolyte & $200 \mu \mathrm{m}$ & {$[13,38]$} \\
\hline \multirow[t]{3}{*}{ Biphasic suspensions } & $\begin{array}{l}\text { Changing inter environment of suspensions with ho- } \\
\text { mopolymer and copolymer, e.g. ionic concentrations }\end{array}$ & $<100 \mu \mathrm{m}$ & [39] \\
\hline & $\begin{array}{l}\text { Controlling the hydrophilicity/hydrophobicity be- } \\
\text { tween particles and solvent }\end{array}$ & - & [39] \\
\hline & Using powder with different isoelectric points (IEP) & - & [39] \\
\hline
\end{tabular}

(a)
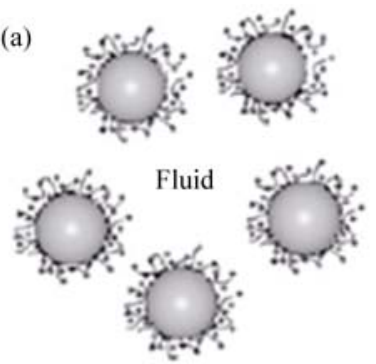

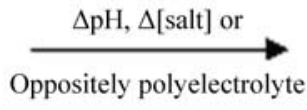

Oppositely polyelectrolyte

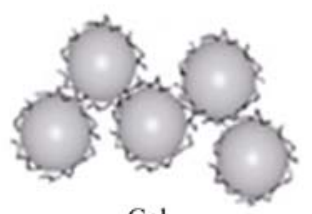

Gel
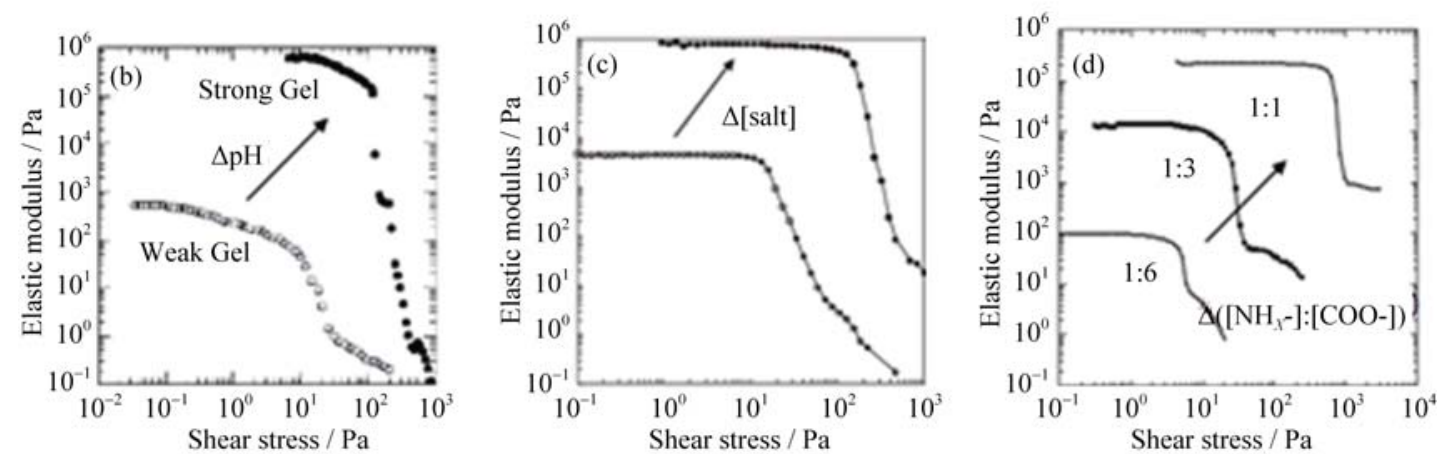

图 3 改变酸碱度、离子强度或者加入反电离聚电解质改变时悬浮液从流体到凝胶的转变

Fig. 3 Fluid-to-gel transition of colloidal inks that occur upon changing $\mathrm{pH}$, ionic strength or adding oppositely charged polyelectrolyte

(a) Schematic view ${ }^{[25,36]}$; (b) Shear elastic modulus versus shear stress for concentrated silica gels of varying strength through changing $\mathrm{pH}^{[25]}$; (c)

Equilibrium elastic modulus of $\mathrm{BaTiO}_{3}$ nanoparticle inks with different salt additions ${ }^{[37]}$; (d) Ink elasticity varying $\left[\mathrm{NH}_{x}^{+}\right]$: $\left[\mathrm{COO}^{-}\right] \mathrm{ratios}^{[38]}$ 
实际上，基于上述自固化悬浮液的设计理论， 调节悬浮液的 $\mathrm{pH}$ 、盐浓度以及加入反离子聚电解质 等均改变颗粒带电量, 降低 Zeta 电位, 增加吸引作 用能 $U$, 使颗粒在引力作用下发生絮凝, 形成由颗粒 相互链接组成的凝胶网络结构, 因此悬浮液的弹性性 能增加并满足自固化悬浮液的要求。Graule 等 ${ }^{[40]}$ 研究 了 $\mathrm{pH}$ 和盐浓度对 $\mathrm{Al}_{2} \mathrm{O}_{3}$ 悬浮液状态的影响。Rhodes 等 ${ }^{[41]}$ 采用激光共聚焦显微镜观察了不同酸碱度和 盐浓度条件下均聚物聚电解质(聚三甲基碘化甲基 丙烯酸乙酯, Poly(trimethylammonium iodide ethyl methacrylate), PTMAM) 分散的二氧化硅悬浮液的微 观结构, 发现随着 $\mathrm{pH}$ 和盐浓度的增加, 均聚物分散 的悬浮液的絮凝程度逐渐增强。此外, $\mathrm{pH}$ 和盐浓度 以及反离子聚电解质的加入可以降低凝胶点固相体 积分数 $\phi_{\mathrm{gel}}$ 。因此, 由式(2)可知, 剪切屈服应力和弹 性模量均会增加。所以, 胶体凝胶悬浮液的线形特 征流体能够很好地保持形状并支撑自身重量, 适于 成型具有跨度的三维周期结构。Smay 等 ${ }^{[25]}$ 通过改 变胶体悬浮液的酸碱度成型 PZT 三维结构, 其特征 尺寸达到了 $100 \mu \mathrm{m}$, 且微观组织均匀细密。

\subsection{2 双相悬浮液}

为了获得更小特征尺寸的三维立体结构, Jennifer 等 ${ }^{[39]}$ 设计了所谓的双相悬浮液(biphasic suspensions)用于直写成型。图 4 所示为双相悬浮液的 制备过程示意图。首先, 制备两种稳定分散的悬浮 液(图 4(a), (b)), 然后将其混合均匀(图 4(c)), 再改变 混合悬浮液的内部条件, 使一种悬浮液内的颗粒发 生絮凝, 而另一种则保持稳定分散, 最终获得由两 相构成的悬浮液(图 4(d))。其中, 一相由溶剂中相互 排斥的胶体颗粒组成, 粘度较低, 能够满足直写成 型的流动性要求; 另一相由溶剂中相互吸引的胶体 颗粒组成, 该相的弹性性能好, 能够满足线形特征 流体弹性性能的要求。

实现双相悬浮液的途径有三种方式：1)两型分 散双相悬浮液。利用均聚物型和共聚物型聚电解

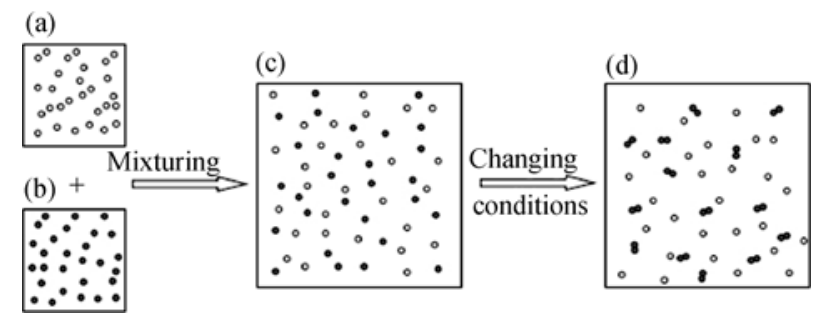

图 4 双相悬浮液的示意图

Fig. 4 Schematic image of biphasic suspensions Two stably dispersed suspensions $(\mathrm{a}, \mathrm{b})$ are mixed $(\mathrm{c})$, and then conditions are changed to trigger the flocculation of one suspension but still another suspensions flowing (d)
质分散剂对离子强度等因素的敏感程度不同来实现: 首先，分别采用均聚物型分散剂(如 PAA)和共聚物型 分散剂(如 PAA-PEO)制备两种分散稳定的悬浮液; 然后，再将两种悬浮液混合均匀; 最后，增加混合 悬浮液的离子强度 ${ }^{[39]}$ 或加入反离子聚电解质 ${ }^{[34]}$ 使 均聚物分散剂的胶体颗粒发生絮凝，而共聚物分散 的胶体颗粒则不因条件的变化而始终保持稳定分散 的状态。2)亲疏水双相悬浮液。此种悬浮液通过亲 水性胶体颗粒、疏水性胶体颗粒和溶剂的亲疏水性 (极性)调控来实现。例如，以亲水性物质(如水)作为 溶剂，那么亲水性胶体颗粒则容易被分散，相互排 斥而稳定分散，而疏水性胶体颗粒则会相互吸引而 絮凝。Jennifer 等 ${ }^{[39]}$ 研究了 $\mathrm{SiO}_{2}$ 的亲疏水双相悬浮 液，其中疏水性的 $\mathrm{SiO}_{2}$ 是采用表面改性方法获得 的。3)等电点双相悬浮液。将等电点不同的两种颗 粒混入相同环境( $\mathrm{pH}$ 、离子强度和分散剂浓度)的溶 剂中，其中一种颗粒在范德华力作用下互相吸引而 絮凝，另一种颗粒则在静电排斥和空间位阻的作用 下相互排斥。除 Jennifer 等的专利之外, 关于等电点 双相悬浮液的研究尚未报道。

\section{3 外固化悬浮的设计}

\section{1 外固化悬浮液的要求与外固化方式}

与自固化悬浮液相同，为了保证直写成型的顺 利实施，外固化悬浮液同样要求具有以下四个特点: 1)通过针嘴微孔时粘度低; 2)通过针孔后在某些特定 的外界条件作用下能够快速固化; 3)固化后的线形特 征体的弹性性能好; 4)悬浮液的固相体积分数较高。 其中，第一点和第四点较容易实现。因为外固化悬浮 液不是依靠自身流变性能的改变来实现固化的，所 以一般稳定分散的悬浮液都能够满足上述要求。第 三点主要受第二点的固化效果影响，因此，第二点 中固化作用条件的设计和控制是其成型的关键。

\section{2 典型的外固化悬浮液}

目前，直写成型外固化悬浮液的固化作用条件 主要有溶剂蒸发、溶解度差异和 UV 固化等三种, 如 表 2 所列 $[17,30-31,42-47]$ 。

\subsection{1 剪切变稀胶体悬浮液}

Cesarano 等 ${ }^{[48]}$ 最先提出了直写成型技术, 并运 用剪切变稀胶体悬浮液进行成型, 他认为用于直写 成型的剪切变稀胶体悬浮液必须满足三个条件：1) 悬浮液为能够顺利通过特殊流体装置(针孔)的假塑 性流体(具有剪切屈服应力的流体，详见[49])；2)从 特殊流体装置(针孔)中出来的悬浮液能够立即失去 
表 2 直写成型的外固化悬浮液 $[17,30-31,42-47]$

Table 2 Assistant-solidification suspensions designed for direct ink writing ${ }^{[17,30-31,42-47]}$

\begin{tabular}{|c|c|c|c|}
\hline $\begin{array}{l}\text { External conditions for } \\
\text { solidification of suspensions }\end{array}$ & Assistant-solidification suspensions & $\begin{array}{l}\text { Minimum feature } \\
\text { size in 3D structures }\end{array}$ & References \\
\hline \multirow{2}{*}{$\begin{array}{l}\text { Fast evaporation of solvent } \\
\text { in suspensions }\end{array}$} & Colloidal suspensions with shear thinning behavior & $500 \mu \mathrm{m}$ & {$[17]$} \\
\hline & Suspensions composited of nanoparticles and organic solvent & $1 \mu \mathrm{m}$ & [42-43] \\
\hline $\begin{array}{l}\text { Solubility divergence between } \\
\text { solvent in suspensions and } \\
\text { liquid in deposition reservoir }\end{array}$ & polyelectrolyte complexes (PECs) & $\begin{array}{l}500 \mu \mathrm{m} \\
30 \mu \mathrm{m} \\
1 \mu \mathrm{m}\end{array}$ & {$[30,43-44]$} \\
\hline \multirow{4}{*}{$\begin{array}{l}\text { Polymerization of organic } \\
\text { monomer under ultraviolet } \\
\text { irradiation }\end{array}$} & Silk fibroin solution & $5 \mu \mathrm{m}$ & {$[45]$} \\
\hline & Sol-Gel suspension & $0.3 \mu \mathrm{m}$ & {$[46]$} \\
\hline & Organic monomer solution & $5 \mu \mathrm{m}$ & {$[31]$} \\
\hline & Suspensions containing organic monomer & - & {$[47]$} \\
\hline
\end{tabular}

流动性; 3 )能够连续成型无缺陷的多层均匀结构。他 主要通过调控固相体积分数来获得假塑性悬浮液 (剪切变稀胶体悬浮液)。该悬浮液的流变性能对固 相体积分数、直写成型的温度和湿度等条件非常敏 感, 极易出现堵嘴等现象。因此, 剪切变稀胶体悬浮 液不是非常适合用于直写成型。

\subsection{2 纳米颗粒/有机溶剂悬浮液}

利用溶剂快速蒸发实现外固化的悬浮液就是采 用有机溶剂制备的含纳米颗粒的悬浮液 (简称, 纳 米颗粒/有机溶剂悬浮液)。该悬浮液采用纳米级的 固体颗粒, 并运用具有保湿作用的高沸点有机溶 剂。因此, 采用此种悬浮液制备的三维结构的特征 尺寸很小, 甚至可达到纳米级。

Ahn 等 ${ }^{[42-43]}$ 采用纳米银/乙二醇悬浮液制备了 最小特征尺寸为 $1 \mu \mathrm{m}$ 的三维结构(高径比值高于 6)。他们以硝酸银为前驱体, 聚丙烯酸为分散剂, 二 乙醇胺为还原剂, 在剧烈摚拌的条件下获得 $5 \mathrm{~nm}$ 左 右的银颗粒, 然后加入乙醇使聚丙烯酸从水中析出 并包㐮在银颗粒表面, 再通过离心提高固相体积分 数, 最后加入有保湿作用的乙二醇来调节固相体积 分数。这种悬浮液可用于制备框架式且具有悬臂结 构的柔性微电极, 对电子封装领域的三维立体封装 具有重大意义。

\subsection{3 溶解差异悬浮液}

除了溶剂蒸发实现固化之外, 利用悬浮液(特 别是有机物质)在溶剂中的溶解度差异也可实现固 化。采用该悬浮液时, 需在特征流体装置(针孔)的下 方放置装有沉积液体的沉积池。当悬浮液从特征流 体装置出来之后, 线形特征流体进入沉积池随即失 去流动性且弹性增加, 从而能够保持其形状。

目前, 依据溶解析出机制, 已用于直写成型是 有机物悬浮液 (或溶液), 聚电解质混合物悬浮液 (polyelectrolyte complexes, PECs) 就是其中的一种。 该悬浮液是将两种带相反电荷的聚电解质的透明水 溶液混合, 聚电解质在静电作用下相互吸引而析出, 形成浑浊的悬浮液, 并且析出物在某些液体中快速 聚集而不分散 ${ }^{[50]}$ 。因此, Gratson 等 ${ }^{[30]}$ 采用聚丙烯酸 和聚乙烯亚胺(PAA-PEI)组成的聚电解质混合物悬 浮液进行直写成型, 并在针孔下方放置装有异丙醇 水溶液的沉积池, 使得直接进入沉积池的线形特征 流体迅速固化。他们系统地 ${ }^{[43]}$ 研究了该聚电解质混 合悬浮液的相行为、流变性能和直写成型。图 5 为 采用沉积池的直写成型示意图 ${ }^{[30]}$ 。他们的研究还表 明, 异丙醇水溶液的浓度对聚电解质混合悬浮液线 形特征流体的固化机制存在影响。当异丙醇的浓度 低于 70vol\%时，固化机制为反聚电解质离子之间强 烈的静电作用机制; 而当异丙醇的浓度高于
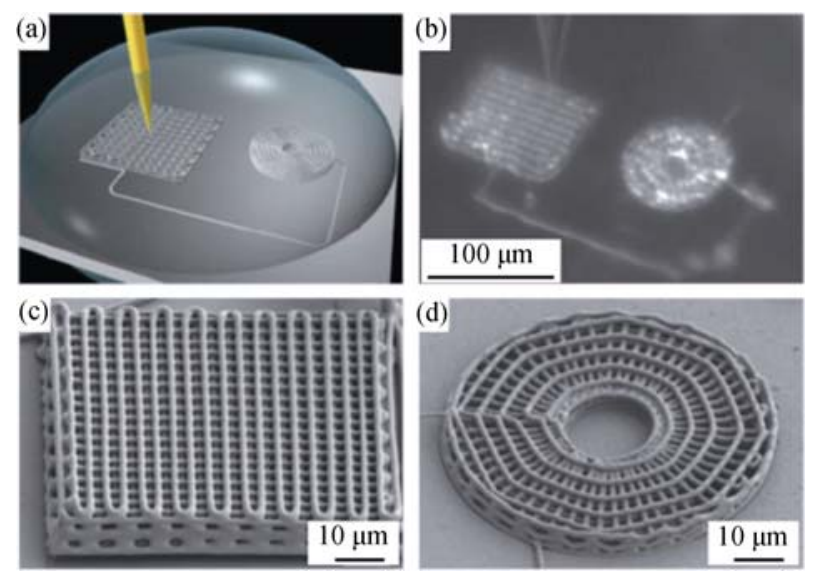

图 5 采用聚电解质混合物制备的三维周期结构

Fig. 5 Direct ink writing of three-dimensional microperiodic structures

(a) Schematic diagram of direct ink writing with deposition reservoir; (b) Optical image acquired in situ during deposition; (c) Three-dimensional periodic structure with a face-centred tetragonal geometry; (d) Threedimensional radial array ${ }^{[30]}$ 
$70 \mathrm{vol} \%$ ，固化机制转变为溶剂的极性作用。Xu 等 ${ }^{[44]}$ 也对由聚丙烯酸和聚烯丙基氯化胺(PAA-PAH)组成 的聚电解质混合悬浮液进行研究, 得出了类似的结 论。Ghosh 等 ${ }^{[45]}$ 将纤维蛋白质溶液用于直写成型, 利 用纤维蛋白质溶液不溶于甲醇水溶液, 快速析出物 能够支持的自身重量的特点, 获得特征尺寸为 $5 \mu \mathrm{m}$ 的三维堆积结构。最优的沉积池液体成分为 $86 \%$ 的 甲醇水溶液。

\subsection{4 溶胶一凝胶悬浮液}

为了避免直写过程中特征线形流体水分的挥发 和成型结构的一致性, 上述悬浮液的直写成型都是 在一个溶剂池(装有与特征流体不润湿的油性液体 ${ }^{[30]}$ 或不溶解且反而析出的沉积液体 ${ }^{[45]}$ )中进行的。实际 上, 如果悬浮液流变性能控制得合适, 直写成型也 能够在空气中直接进行。当线形特征流体尺寸非常 小达到微米级甚至纳米级时, 线形流体的比表面积 将非常大。此时, 溶剂挥发迅速, 悬浮液的流变性能 也会发生巨大的转变。Duoss 等 ${ }^{[46]}$ 估算了直径为 $1 \mu \mathrm{m}$ 的线形特征流体的 “固化” 时间为 $1 \mathrm{~ms}$, 推断流体 经过 $4 \mu \mathrm{m}$ 间隔的速度达到 $400 \mu \mathrm{m} / \mathrm{s}$ 就可进行直写 成型。因此, Duoss 等 ${ }^{[46]}$ 设计了一种新型溶胶-凝胶 悬浮液。他们以无水乙醇为溶剂, 亚乙酰丙酮一二异 丙氧基钛为前驱体, 在 $80^{\circ} \mathrm{C}$ 下通过金属醇盐的水解 和缩合使溶剂由溶胶转变为凝胶, 然后依靠有机溶 剂的挥发来实现固化。实际上, 该悬浮液为有机金 属盐凝胶态溶液, 内部并无悬浮固体颗粒, 因此它 非常容易通过针嘴微孔, 获得的周期结构的最小特 征尺寸也很小。Duoss 等 ${ }^{[46]}$ 成型的三维周期结构平 整、尺寸均匀, 最小特征尺寸约为 $0.3 \mu \mathrm{m}$ 。 Ahn 等 ${ }^{[51]}$ 设计了可用于制备 ITO 微电极的溶胶一凝胶悬浮液, 他们将醋酸铟和乙酰丙酮氯化锡加入乙酰丙酮中, 并添加四甲基氢氧化铵(TMAH, tetramethylammonium hydroxide)和双氧水使之氧化, 最后获得可直 写成型的 ITO 溶胶-凝胶悬浮液。

\subsection{5 有机聚合型悬浮液}

有机聚合型悬浮液中含有在外界条件(如紫外 光或微波等)作用下能聚合的有机物, 可以通过外 界条件引发聚合实现成型固化。该成型过程也需要 在特征流体出口(针尖)处加装可控制外界条件的装 置。孙竞博等 ${ }^{[52-54]}$ 以甲基丙烯酸甲酯为单体, 季戊四 醇三丙稀酸脂为交联剂, 2, 2-二乙氧基苯乙酮为光引 发剂, 配制 $\mathrm{BaTiO}_{3}$ 的光固化型悬浮液, 并在配备紫 外光源的点胶系统上制备出特征尺寸为 $0.4 \mathrm{~mm}$ 的三 维结构。此前, Barry 等 ${ }^{[31]}$ 利用相同原理制备了三维 水凝胶堆积结构, 并用于控制细胞生长。

\section{4 结束语}

直写成型技术是一种新型的三维复杂结构的制 备方法, 已经用于微电子封装、锂离子电池、光子 晶体和生物医用材料等领域。直写成型用悬浮液有 以下几个发展方向:

1) 优化使颗粒间相互作用能 (即范德华引力作 用能 $U_{\mathrm{vdW}}$ 、静电排斥作用能 $U_{\text {elect }}$ 和空间位阻作用 能 $U_{\text {steric }}$ 改变的某些途径, 从而调节悬浮液流变性 能, 实现自固化型的直写成型。改变悬浮液的 $\mathrm{pH}$ 和 盐浓度, 以及添加与分散剂类型相反的聚电解质是 自固化直写成型悬浮液的三种常见方式。但此种悬 浮液的工艺参数范围窄, 其流变性能难控制。双相 悬浮液甚至多相悬浮液是更好的方式。例如, 利用 均聚物型和共聚物型分散剂对离子浓度敏感性的差 异可制备一种双相悬浮液。但是针对此种双相悬浮 液的分散剂(均聚物和共聚物的种类、构型与分子量) 的合成和研究尚待进行, 两类分散剂对离子浓度敏 感性的差异以及评价手段也尚未实施。此外, 通过 粉体表面改性可以得到亲疏水性或等电点不同的粉 体的双相悬浮液, 但是相关工作仅在 Jennifer 的专 利中提到。

2) 开发改变颗粒间相互作用能(耗散作用能 $\left.U_{\text {depletion}}\right)$ 的其他方法, 进而控制悬浮液流变性能进 行直写成型。例如, 调控游离态聚合物、聚电解质 与细小胶体颗粒等耗散物质及其数量。相关研究也 尚未见报道。

3) 除了进一步完善已有的外固化控制方式之 外, 还需研究更易控的外界控制方法进行直写成 型。例如，通过磁场或电场控制悬浮液内溶解的智 能有机物形态等。与自固化悬浮液相比, 此类方式 能更为方便和稳定的控制悬浮液流变性能, 所获得 的三维结构的特征尺寸也更为细小。

\section{参考文献:}

[1] LEWIS J A. Colloidal processing of ceramics. Journal of the American Ceramic Society, 2000, 83(10): 2341-2359.

[2] LEWIS J A, GRATSON G M. Direct writing in three dimensions. Materials Today, 2004, 7(7): 32-39.

[3] LEWIS J A, SMAY J E, STUECKER J, et al. Direct ink writing of three-dimensional ceramic structures. Journal of the American Ceramic Society, 2006, 89(12): 3599-3609.

[4] ALLAHVERDI M, DANFORTH S C, JAFARI M, et al. Processing of advanced electroceramic components by fused deposition 
technique. Journal of the European Ceramic Society, 2001, 21(10): 1485-1490.

[5] SAFARI A, AKDOGAN E K. Rapid prototyping of novel piezoelectric composites. Ferroelectrics, 2006, 331(1): 153-179.

[6] TRESSLER J F, ALKOY S, DOGAN A, et al. Functional composites for sensors, actuators and transducers. Composites. Part A: Applied Science and Manufacturing, 1999, 30(4): 477-482.

[7] LI Y, LI L, LI B. Direct ink writing of 3-3 piezoelectric composite. Journal of Alloys and Compounds, 2015, 620(25): 125-128.

[8] SAN MARCHI C, KOUZELI M, RAO R, et al. Alumina-aluminum interpenetrating-phase composites with three-dimensional periodic architecture. Scripta Materialia, 2003, 49(9): 861-866.

[9] RAO M P, SANCHEZ-HERENCIA A J, BELTZ G E, et al. Laminar ceramics that exhibit a threshold strength. Science, 1999, 286(5437): 102-105.

[10] SOUNDARARAJAN R, KUHN G, ATISIVAN R, et al. Processing of mullite-aluminum composites. Journal of the American Ceramic Society, 2001, 84(3): 509-513.

[11] EQTESADI S, MOTEALLEH A, MIRANDA P, et al. Robocasting of 45S5 bioactive glass scaffolds for bone tissue engineering. Journal of the European Ceramic Society, 2014, 34(1): 107-118.

[12] MAAZOUZ Y, MONTUFAR E B, GUILLEM-MARTI J, et al. Robocasting of biomimetic hydroxyapatite scaffolds using self-setting inks. Journal of Materials Chemistry B, 2014, 2(33): $5378-5386$.

[13] MICHNA S, WU W, LEWIS J A. Concentrated hydroxyapatite inks for direct-write assembly of 3-D periodic scaffolds. Biomaterials, 2005, 26(28): 5632-5639.

[14] JOANNOPOUlos J D, VILLENEUVE P R, FAN S. Photonic crystals: putting a new twist on light. Nature, 1997, 386(6621): $143-149$.

[15] SHEN W, LI M, YE C, et al. Direct-writing colloidal photonic crystal microfluidic chips by inkjet printing for label-free protein detection. Lab on a Chip, 2012, 12(17): 3089-3095.

[16] VLASOV Y A, BO X Z, STURM J C, et al. On-chip natural assembly of silicon photonic bandgap crystals. Nature, 2001, 414(6861): 289-293.

[17] CESARANO III J. A review of robocasting technology. Materials Research Symposium Proceedings, 1999, 542: 133-139.

[18] CESARANO III J, BAER T A, CALVERT P. Recent Developments in Freeform Fabrication of Dense Ceramics from Slurry Deposition. Albuquerque, NM: Sandia National Labs., 1997, SAND97-2857C.

[19] ZEIN I, HUTMACHER D W, TAN K C, et al. Fused deposition modeling of novel scaffold architectures for tissue engineering applications. Biomaterials, 2002, 23(4): 1169-1185.

[20] AGARWALA M K, BANDYOPADHYAY A, WEEREN R, et al. FDC, rapid fabrication of structural components. American Ce- ramic Society Bulletin, 1996, 75(11): 60-65.

[21] RANGARAJAN S, QI G, VENKATARAMAN N, et al. Powder processing, rheology, and mechanical properties of feedstock for fused deposition of $\mathrm{Si}_{3} \mathrm{~N}_{4}$ ceramics. Journal of the American Ceramic Society, 2000, 83(7): 1663-1669.

[22] SACHS E, CIMA M, WILLIAMS P, et al. Three dimensional printing: rapid tooling and prototypes directly from a CAD model. Journal of Manufacturing Science and Engineering, 1992, 114(4): $481-488$.

[23] LEE K W, WANG S, LU L, et al. Fabrication and characterization of poly (propylene fumarate) scaffolds with controlled pore structures using 3-dimensional printing and injection molding. Tissue Engineering, 2006, 12(10): 2801-2811.

[24] SACHS E, CIMA M, BREDT J, et al. CAD-casting: direct fabrication of ceramic shells and cores by three-dimensional printing. Manufacturing Review (USA), 1992, 5(2): 117-126.

[25] SMAY J E, CESARANO J, LEWIS J A. Colloidal inks for directed assembly of 3-D periodic structures. Langmuir, 2002, 18(14): 5429-5437.

[26] AHN B Y, SHOJI D, HANSEN C J, et al. Printed origami structures. Advanced Materials, 2010, 22(20): 2251-2254.

[27] LI QI, LI BO, ZHOU JI, et al. Robocasting: a novel avenue for engineering complex 3D structures. Journal of Inorganic Materials, 2005, 20(1): 13-20.

[28] CESARANO J, SEGALMAN R, CALVERT P. Robocasting provides moldless fabrication from slurry deposition. Ceramic Industry, 1998, 148(4): 94-102.

[29] STUECKER J N, CESARANO J, HIRSCHFELD D A. Control of the viscous behavior of highly concentrated mullite suspensions for robocasting. Journal of Materials Processing Technology, 2003, 142(2): 318-325.

[30] GRATSON G M, XU M, LEWIS J A. Microperiodic structures: direct writing of three-dimensional webs. Nature, 2004, 428(6981): 386.

[31] BARRY R A, SHEPHERD R F, HANSON J N, et al. Direct-write assembly of 3D hydrogel scaffolds for guided cell growth. Advanced Materials, 2009, 21(23): 2407-2410.

[32] HERSCHEL W H, BULKLEY R. Konsistenzmessungen von gummi-benzollösungen. Kolloid-Zeitschrift, 1926, 39(4): 291-300.

[33] CHANNELL G M, ZUKOSKI C F. Shear and compressive rheology of aggregated alumina suspensions. AIChE Journal, 1997, 43(7): 1700-1708.

[34] TRAPPE V, PRASAD V, CIPELLETTI L, et al. Jamming phase diagram for attractive particles. Nature, 2001, 411(6839): 772-775.

[35] SMAY J E, CESARANO J, TUTTLE B A, et al. Directed colloidal assembly of linear and annular lead zirconate titanate arrays. Journal of the American Ceramic Society, 2004, 87(2): 293-295. 
[36] SMAY J E, CESARANO III J, TUTTLE B A, et al. Piezoelectric properties of $3-\mathrm{X}$ periodic $\mathrm{Pb}\left(\mathrm{Zr}_{X} \mathrm{Ti}_{1-x}\right) \mathrm{O}_{3}$-polymer composites. Journal of Applied Physics, 2002, 92(10): 6119-6127.

[37] LI Q, LEWIS J A. Nanoparticle inks for directed assembly of three-dimensional periodic structures. Advanced Materials, 2003, 15(19): 1639-1643.

[38] RAO R B, KRAFCIK K L, MORALES A M, et al. Microfabricated deposition nozzles for direct-write assembly of three-dimensional periodic structures. Advanced Materials, 2005, 17(3): 289-293.

[39] LEWIS J A, LI Q, RAO R. Biphasic Inks. U.S. Patent 8187500. 2012-5-29.

[40] GRAUlE T J, GAUCKLER L J, BAADER F H. Direct coagulation casting-a new green shaping technique PT. 1. processing principles. Industrial Ceramics, 1996, 16(1): 31-34.

[41] RHODES S K, LAMBETH R H, GONZALES J, et al. Cationic comb polymer superdispersants for colloidal silica suspensions. Langmuir, 2009, 25(12): 6787-6792.

[42] AHN B Y, DUOSS E B, MOTALA M J, et al. Omnidirectional printing of flexible, stretchable, and spanning silver microelectrodes. Science, 2009, 323(5921): 1590-1593.

[43] GRATSON G M, LEWIS J A. Phase behavior and rheological properties of polyelectrolyte inks for direct-write assembly. Langmuir, 2005, 21(1): 457-464.

[44] XU M, LEWIS J A. Phase behavior and rheological properties of polyamine-rich complexes for direct-write assembly. Langmuir, 2007, 23(25): 12752-12759.

[45] GHOSH S, PARKER S T, WWANG X, et al. Direct-write assembly of microperiodic silk fibroin scaffolds for tissue engineering applications. Advanced Functional Materials, 2008, 18(13):
1883-1889.

[46] DUOSS E B, TWARDOWSKI M, LEWIS J A. Sol-Gel inks for direct-write assembly of functional oxides. Advanced Materials, 2007, 19(21): 3485-3489.

[47] SHEPHERD R F, PANDA P, BAO Z, et al. Stop-flow lithography of colloidal, glass, and silicon microcomponents. Advanced Materials, 2008, 20(24): 4734-4739.

[48] CESARANO III J, KING B H, DENHAM H B. Recent Developments in Robocasting of Ceramics and Multimaterial Deposition. Albuquerque, NM: Sandia National Labs., 1998, SAND98-2195C.

[49] 高 濂, 孙 静, 刘阳桥. 纳米粉体的分散及表面改性. 北京: 化 学工业出版社, 2003: 65-90.

[50] PHILIPP B, DAUTZENBERG H, LINOW K J, et al. Polyelectrolyte complexes-recent developments and open problems. Progress in Polymer Science, 1989, 14(1): 91-172.

[51] AHN B Y, LORANG D J, DUOSS E B, et al. Direct-write assembly of microperiodic planar and spanning ITO microelectrodes. Chemical Communications, 2010, 46(38): 7118-7120.

[52] SUN JING-BO, LI BO, HUANG XUE-GUANG, et al. Direct-write assembly of ceramic three-dimensional structures based on photosensitive inks. Journal of Inorganic Materials, 2009, 24(6): $1147-1150$

[53] SUN JING-BO, LI BO, CAI KUN-PENG, et al. $\mathrm{TiO}_{2}$ photolysis device fabricated by direct ink write assembly. Journal of Inorganic Materials, 2011, 26(3): 300-304.

[54] CAI KUN-PENG, LI YA-YUN, SUN ZHEN-XIN, et al. Preparation of $3 \mathrm{D}$ ceramic meshes by direct-write method and modulation of its photocatalytic properties by structure design. Journal of Inorganic Materials, 2012, 27(1): 1-5. 